Pacific Journal of Mathematics

EXISTENCE THEOREMS FOR CERTAIN QUASI-LINEAR 


\section{EXISTENCE THEOREMS FOR CERTAIN QUASI-LINEAR ELLIPTIC EQUATIONS}

\section{ZANe C. MotTeleR}

This paper is devoted to proving the existence of solutions (in the classical sense) for a certain Dirichlet problem in the theory of quasi-linear elliptic partial differential equations of the second order. The principal equation considered is one which can be written in the form

$$
\frac{\partial}{\partial x_{k}}\left[a_{k}(\nabla \varphi)\right]=\frac{\partial a_{k}(\nabla \varphi)}{\partial \varphi_{x_{l}}} \frac{\partial^{2} \varphi}{\partial x_{k} \partial x_{l}}=a_{k l}(\nabla \varphi) \varphi_{x_{k} x_{l}}=g(x, \varphi, \nabla \varphi) .
$$

If the matrix $\left(a_{k l}\right)$ is positive definite, if the functions $a_{k l}$ and $g$ are Hölder continuous in all arguments, and if the ratio of $|g|$ to the minimum eigenvalue of $\left(a_{k l}\right)$ grows less rapidly than the first power of $|\nabla \varphi|$ for large $|\nabla \varphi|$, then the Dirichlet problem for $\varphi$ satisfying the above equation with its values given on the sufficiently smooth boundary of a bounded domain has a solution.

The general quasi-linear partial differential equation of second order in $n$ variables is $^{1}$

$$
a_{k l}\left(x, \varphi, \varphi_{x}\right) \varphi_{k l}+a\left(x, \varphi, \varphi_{x}\right)=0,
$$

where $x$ and $\varphi_{x}$ represent the vectors with components $\left(x_{1}, \cdots, x_{n}\right)$ and $\left(\varphi_{1}, \cdots, \varphi_{n}\right)$ respectively. We shall use the notations

$$
\varphi_{k}=\varphi_{x_{k}}=\frac{\partial \varphi}{\partial x_{k}} \text { and } \varphi_{k l}=\varphi_{x_{k} x_{l}}=\frac{\partial^{2} \varphi}{\partial x_{k} \partial x_{l}}
$$

extensively. For (1) to be elliptic, the matrix of coefficients $\left(a_{k l}\right)$ must be positive definite, i.e., its minimum eigenvalue must be positive. Throughout this paper we shall assume this to be the case, and that, furthermore, equation (1) can be written in divergence form, i.e.,

$$
\frac{\partial}{\partial x_{k}}\left[a_{k}\left(x, \varphi, \varphi_{x}\right)\right]+a\left(x, \varphi, \varphi_{x}\right)=0 \text {. }
$$

This class of equations includes most of the equations of importance in applications, namely those representing conservation laws or variational principles.

Received November 20, 1964. This paper embodies the major portion of the author's doctoral dissertation for Stanford University. Part of this work was done under the auspices of the Atomic Energy Commission.

1 We shall always assume summation from 1 to $n$ over repeated indices. 
The recent paper of Ladyzhenskaya and Ural'tseva [7] explores the existence problem for (2) in detail. In this work the authors consider problems in which the ellipticity hypothesis takes the form

$0<C_{1}\left(1+|p|^{2}\right)^{(m-2) / 2}|\xi|^{2} \leqq \alpha_{k l}(x, \varphi, p) \xi_{k} \xi_{l} \leqq C_{2}\left(1+|p|^{2}\right)^{(m-2) / 2}|\xi|^{2}, m>1$,

for all real nonzero $n$-vectors $\left(\xi_{1}, \cdots, \xi_{n}\right)^{2}$. Besides this the authors assume that the coefficients $a_{k l}(x, \varphi, p)$ and $a(x, \varphi, p)$ behave roughly like powers of $|p|$ for large $|p|$, which power does not decrease when the $a_{k l}$ and $a$ are differentiated with respect to $x$ or $\varphi$, but which decreases by at least one under differentiation by $p$. Using these and certain other simplifying assumptions, the authors establish a number of interesting existence theorems. Morrey [8] and Stampacchia [14] have also recently considered quasi-linear elliptic partial differential equations in $n$ variables, usually in connection with variational problems, in which the coefficients likewise satisfy power growth properties in $|p|$.

On the other hand, using no power growth assumptions at all, Gilbarg [6] has proved the existence of a solution to the boundary value problem

$$
\frac{\partial}{\partial x_{k}}\left[a_{k}\left(\varphi_{x}\right)\right]=0 \text { in } \Omega, \varphi=f \text { on } \partial \Omega
$$

where $f$ is twice continuously differentiable and $\Omega$ is a bounded domain with sufficiently smooth boundary $\partial \Omega$. Only ellipticity and either the boundedness of the ratio of the largest and smallest eigenvalues of the coefficient matrix, or the strict convexity of the domain $\Omega$, are assumed.

In this paper we shall prove existence theorems for (2) under the weakened ellipticity hypothesis of Gilbarg, as well as in the nonuniformly elliptic case, thus extending and generalizing the work of Gilbarg and Ladyzhenskaya-Ural'tseva. A few additional hypotheses are necessitated by the more general form of the problem, but in many cases the existence theorem does not hold if the hypotheses are weakened, and when this is the case, it will be pointed out with appropriate counterexamples.

2. Statement of the main theorems, Let a bounded domain $\Omega$ be given in Euclidean $n$-space and be fixed for the remainder of the discussion. Assume that its boundary $\partial \Omega$ is sufficiently smooth for our purposes; this will be made more precise later. We shall first consider the following boundary value problem:

${ }^{2}$ We shall often use " $p$ " to represent " $\varphi_{x}$ " where convenient without confusion. The notation "|.|" will mean the Euclidean norm or one of its equivalents. 


$$
\begin{gathered}
\frac{\partial}{\partial x_{k}}\left[\alpha_{k}\left(\varphi_{x}\right)\right]=g\left(x, \varphi, \varphi_{x}\right) \text { in } \Omega ; \\
\varphi=0 \text { on } \partial \Omega .
\end{gathered}
$$

We shall assume that the $a_{k}$ possess Hölder continuous first partial derivatives and that $g$ is Hölder continuous in all arguments. The coefficients

$$
a_{k l}(p)=\frac{\partial a_{k}(p)}{\partial p_{l}}
$$

will satisfy one of the following hypotheses, where $\xi=\left(\xi_{1}, \cdots, \xi_{n}\right)$ is a real, nonzero $n$-vector:

I. Uniform Ellipticity.

$$
0<\lambda_{1}(p)|\xi|^{2} \leqq a_{k l} \xi_{k} \xi_{l} \leqq \lambda_{2}(p)|\xi|^{2} ; \frac{\lambda_{2}}{\lambda_{1}}(p) \leqq \gamma<\infty
$$

II. Non-uniform Ellipticity. $0<\lambda_{1}(p)\left\{\left.\xi\right|^{2} \leqq a_{k l}(p) \xi_{k} \xi_{l} ; \Omega\right.$ strictly convex. By continuity $\lambda_{1}$ and $\lambda_{2}$ cannot approach zero or infinity unless $|p| \rightarrow \infty$.

The following theorem, the proof of which is carried out in $\S 3$, is the principal result of this paper.

THEOREM 1. Let either Hypothesis I or II be satisfied. If there exist positive functions $\lambda(|p|)$ and $G(|p|)$ which are, respectively, monotone decreasing and monotone increasing in $|p|$, with $\lambda_{1}(p) \geqq$ $\lambda(|p|)$ and $|g(x, \varphi, p)| \leqq G(|p|)$ uniformly in $x$ and $\varphi$, such that ${ }^{3}$ $G(|p|) / \lambda(|p|) \leqq C|p|^{\alpha}(\alpha<1)$ for sufficiently large $|p|$, then problem (3), (4) has a solution.

In $\S 4$ we shall explore, by means of examples, borderline cases of the hypotheses of Theorem 1 and find that for the most part any relaxation of hypotheses will lead to problems which do not, in general, possess solutions. Note that the hypotheses call for a function $g(x, \varphi, p)$ on the right side which is majorized by a function $G(|p|)$ uniformly in $x$ and $\varphi$. For such a majorant to exist, it is sufficient, in general, that one be able to find an a priori bound on $\varphi$, that is, to show that there is a constant $M$ depending only on the given constants, namely

${ }^{3}$ A more general statement of this is that the integral $\int_{R}^{M} \frac{\lambda(\xi+M)}{G(\xi+M)} d \xi$ should become unbounded as $M \rightarrow \infty$. The latter will be used in the proof of the theorem, and the simpler statement for the purpose of formulating examples, etc. 
the volume of $\Omega, \gamma, C$, and $\alpha$, but independent of the particular solution $\varphi$, such that $|\varphi| \leqq M$ for any solution $\varphi$ of (3), (4). Some conditions under which such a bound can be found are enumerated and explained in $\S 5$.

When $g$ is a function of $x$ and $\varphi$ alone, Theorem 1 can be restated as follows:

CoRollaRY. Let the coefficients of (3) satisfy either I or II. Furthermore, for sufficiently large $|p|$, assume $\lambda_{1}(p) \geqq C /|p|^{\alpha}$ for some $\alpha<1$. If it is known a priori that any solution to (3), (4) is bounded by a fixed constant independent of the particular solution, then problem (3), (4) indeed has a solution.

This corollary includes as a special case a theorem of Stampacchia (cf. [14], Theorem 10.2). Stampacchia's theorem holds for the nonuniformly elliptic case when in addition there is a function $f(p)$ with $a_{k}(p)=\partial f(p) / \partial p_{k}$, so that (3) is the Euler equation for a variational problem, and when certain additional hypotheses on power dependence of $f$ and its derivatives, enumerated in condition $6^{\circ}$ of $\S 5$ of this paper, are satisfied.

In the above corollary, because $\varphi_{x}$ is not among the arguments of $g$, we can find a solution whenever we can bound $\varphi . \$ 5$ considers various conditions on $g$ which will ensure the existence of such a bound. The lack of dependence on $\varphi_{x}$ makes it possible to find new conditions which do not suffice in the general case.

Next we consider a second class of problems:

$$
\frac{\partial}{\partial x_{k}}\left[a_{k}\left(x, \varphi_{x}\right)\right]=0 \text { in } \Omega, \varphi=0 \text { on } \partial \Omega \text {. }
$$

After the indicated differentiations have been performed, this equation is also inhomogeneous, and a result analogous to Theorem 1 can be stated for it:

THEOREM 2. Let $a_{k l}(x, p)=\partial a_{k}(x, p) / \partial p_{l}$ satisfy Hypothesis $I$ or II. If $\sum_{i, k}\left|\partial a_{k}(x, p) / \partial x_{i}\right| \leqq A(|p|)$ where $A(|p|)$ is monotone increasing in $|p|$, and if there exists a monotone decreasing positive function $\lambda(|p|) \leqq \lambda_{1}(p)$ such that $t^{4} A(|p|) / \lambda(|p|) \leqq C|p|^{\alpha}(\alpha<1)$ for sufficiently large $|p|$, then a solution to problem (5) exists.

${ }^{4}$ Or, as in Theorem $1, \lim _{M \rightarrow \infty} \int_{h}^{M} \frac{\lambda(\xi+M)}{A(\xi+M)} d \xi=+\infty$. 
Theorems 1 and 2 can now be combined to yield an existence theorem for problems of the form

$$
\frac{\partial}{\partial x_{k}}\left[a_{k}\left(x, \varphi_{x}\right)\right]=g\left(x, \varphi, \varphi_{x}\right) \text { in } \Omega, \varphi=0 \text { on } \partial \Omega .
$$

This theorem can be stated in the same terms as Theorem 2, except that we replace

$$
\sum_{i, k}\left|\frac{\partial a_{k}(x, p)}{\partial x_{i}}\right| \text { by } \sum_{i, k}\left|\frac{\partial a_{k}(x, p)}{\partial x_{i}}\right|+|g(x, \varphi, p)|
$$

where it occurs. This result will be referred to hereafter as Theorem 3.

If, now, we attempt the further generalization of allowing $\phi$ among the arguments of the $a_{k}$ in (6), the problem is greatly complicated, and one must make further restrictions on the $a_{k}$ and their derivatives to assure the existence of solutions. This further generalization will not be treated here.

Before proving these theorems, let us briefly consider the subject of uniqueness of solutions of (6), which of course includes the previous cases also.

Definition. A function $h(x)$ which satisfies the condition

$$
|h(x+y)-h(x)| \geqq f(y) \text { with } \int_{0}^{1} \frac{d y}{f(y)}=\infty,
$$

where $f$ is such that $f^{\prime}(y) \geqq A, A$ being a constant, is said to satisfy an Osgood condition.

Theorem 4. (Uniqueness). If all the hypotheses of Theorem 3 are satisfied, and if, in addition, $g(x, \varphi, p)$ and the $a_{k l}(x, p)$ and $\partial a_{k}(x, p) / \partial x_{i}$ all satisfy Osgood conditions in the $p$ variables with the same function $f$, and if $g$ is nondecreasing in $\varphi$ for all $x$ and $p$, then (6) has at most one solution.

This result is due to Redheffer ([11], Satz I), who also showed ([10], pp. 36ff) that the Osgood condition is necessary.

3. Proofs and consequences of the main theorems. In this section we shall prove a series of lemmas which, taken together, will establish Theorem 1. These lemmas are in the nature of a priori bounds on possible solutions and their derivatives. Using these bounds, 
one obtains existence by application of the Leray-Schauder fixed point theorem. First we need the following:

Remark. Consider the problem

$$
\begin{gathered}
\frac{\partial}{\partial x_{k}}\left[a_{k}\left(\varphi_{x}\right)\right]=g\left(x, \varphi, \varphi_{x}\right) \text { in } \Omega ; \\
\varphi=0 \text { on } \partial \Omega,
\end{gathered}
$$

where all the hypotheses of Theorem 1 are satisfied. Then there exists a constant $M=M(C, \alpha)$, independent of $\varphi$ so that $|\varphi| \leqq M$ for all solutions of (7), (8).

Proof. The ratio $|g(x, \varphi, p)| / M_{2} \Sigma\left|p_{i}\right| \alpha_{i i}(p) \leqq G(|p|) / M_{2}|p| \lambda(|p|)$ can be made less than unity for all $\left|p_{i}\right|>M_{2}$, for sufficiently large $M_{2}$ (depending on $C$ and $\alpha$ ). This implies the desired bound on $|\varphi|$, for define $\psi=-M_{1} \sum_{i} e^{-M_{2} x_{i}}$, where $M_{1}=\max e^{M_{2} x_{i}}$. If $\varphi(x)-\psi(x)$ attains its maximum at some $x_{1}$ inside $\Omega$, then at $x_{1}, \varphi_{x_{i}}=$ $\psi_{x_{i}}(i=1,2, \cdots, n)$ and $\alpha_{i j}\left(\varphi_{x}\right) \varphi_{i j} \leqq \alpha_{i j}\left(\varphi_{x}\right) \psi_{i j}$; but $\alpha_{i j}\left(\varphi_{x}\right) \varphi_{i j}=g\left(x_{1}, \varphi, \varphi_{x}\right)=$ $g\left(x_{1}, \varphi, \psi_{x}\right)$; hence $g\left(x_{1}, \varphi, \psi_{x}\right) \leqq a_{i j} \psi_{i j}=-M_{2}^{2} M_{1} \sum_{i} a_{i i}\left(\psi_{x}\right) e^{-M_{2} x_{i}}=$ $-M_{2} \Sigma a_{i i}\left|\psi_{i}\right|$. This contradicts the assertion $|g(x, \varphi, p)| / M_{2} \Sigma\left|p_{i}\right| a_{i i}(p)<$ 1 , since $\psi_{k}=M_{1} M_{2} e^{-M_{2} x_{k}} \geqq M_{2}$. Hence $x_{1} \in \partial \Omega$ and we conclude that $\varphi(x)-\psi(x) \leqq-\psi\left(x_{1}\right)$, which gives an upper bound on $\varphi(x)$. Considering $\varphi(x)+\psi(x)$ where it has a minimum, we obtain similarly a lower bound for $\varphi$. This proof is due to Ladyzhenskaya-Ural'tseva ([7], p. 79).

LEMma 1. Let the coefficients of (7) satisfy all the hypotheses of Theorem 1. Then there exists a constant ${ }^{5} K=K(\max |\varphi|, C, \alpha, \gamma ; \Omega)$ independent of $\varphi$ such that $\max _{\partial \Omega}|\nabla \phi| \leqq K$ for all solutions $\phi$ of (7), (8).

Proof. Following Ladyzhenskaya-Ural'tseva ([7], p. 40) we shall construct two functions $\psi(x)$ and $w(x)$ to aid us in estimating $|\nabla \phi|$. Let $\varphi$ and $\psi$ be related by the equation $\varphi=f(\psi)$. Then $\varphi_{i}=f^{\prime} \psi_{i}$ and $\varphi_{i j}=f^{\prime \prime} \psi_{i} \psi_{j}+f^{\prime} \psi_{i j}$. Hence (7) yields

$$
a_{i j} \psi_{i j}+\frac{f^{\prime \prime}}{f^{\prime}} a_{i j} \psi_{i} \psi_{j}=\frac{g}{f^{\prime}}
$$

Assume for the moment that $f$ can be chosen so that $f^{\prime \prime} \mid f^{\prime}>0$ and $f^{\prime}>0$. Then case.

${ }^{5}$ In this and what follows, dependence on $\gamma$ occurs only in the uniformly elliptic 
(9)

$$
\begin{aligned}
a_{i j} \psi_{i j} & =-\frac{f^{\prime \prime}}{f^{\prime}} a_{i j} \psi_{i} \psi_{j}+\frac{g}{f^{\prime}} \geqq \\
& -\frac{f^{\prime \prime}}{f^{\prime}} \lambda_{1}|\nabla \psi|^{2}-\frac{|G|}{f^{\prime}} \geqq \lambda_{1}\left\{-\frac{f^{\prime \prime}}{f^{\prime}}|\nabla \psi|^{2}-\frac{C|\nabla \varphi|^{\alpha}}{f^{\prime}}\right\} .
\end{aligned}
$$

Let a constant ${ }^{6} C$ be chosen so that $|\nabla \varphi|^{\alpha} \leqq C\left(|\nabla \varphi|^{2}+1\right)$. Then putting this expression into (9), we obtain

$$
a_{i j} \psi_{i j} \geqq \lambda_{1}\left[|\nabla \psi|^{2}\left(-\frac{f^{\prime \prime}}{f^{\prime}}-C f^{\prime}\right)-\frac{C}{f^{\prime}}\right]
$$

It is possible to choose $f$ so that the quantity in square brackets is bounded from below by a constant $C_{1}$; for choose $f$ such that

$$
\varphi=f(\psi)=\frac{1}{C} \ln (1+\psi)
$$

Then $-f^{\prime \prime} \mid f^{\prime}-C f^{\prime}=0, f(0)=0$, and $\psi=-1+e^{\partial \varphi}>-1$; in fact $\psi$ is bounded away from -1 and $\infty$ in terms of $\max |\varphi|$. Hence we can choose $C_{1}=-C / \operatorname{Min} f^{\prime}$.

Our next task is to construct a function $w(x)$ such that $a_{i j}\left(w_{i j}+\psi_{i j}\right)>0$, and $w(x)$ takes its maximum at some point $q \in \partial \Omega$. First we choose a twice continuously differentiable function $u(x)$ such that

(i) $u(x)>0$ in $\Omega$;

(ii) $|\nabla u| \geqq$ const. $>0$;

(iii) $u(x)=0$ at $q$. Such a function exists if $\partial \Omega$ is sufficiently reasonable; for instance, if Hypothesis $I$ is satisfied, and if $\partial \Omega$ is such that for each $q \in \partial \Omega$ there exists a sphere of radius $\rho$ (constant), the closure of which intersects $\bar{\Omega}$ only at $q$, then we can take $u(x)=$ $1 / \rho-1 / r$ where $r$ is the distance of $x$ from the center of the circle. If Hypothesis II is satisfied, $u(x)$ can be taken to be the function which measures the distance to $x$ from a plane intersecting $\partial \Omega$ only at $q$. Since $\Omega$ is strictly convex, such a plane exists for each $q \in \partial \Omega$ and $u(x)>0$ throughout $\Omega$. Now define

$$
w(x)=m e^{-a u(x)} \text {. }
$$

Clearly $w$ assumes its maximum $m$ in $\bar{\Omega}$ at $q$, and a simple calculation using the specific functions $u$ mentioned above shows that the constants $a$ and $m$ can be chosen so that

$$
\frac{a_{i j} w_{i j}}{\lambda_{1}}=m\left(-a \frac{a_{i j}}{\lambda_{1}} u_{i j}+a^{2} \frac{a_{i j}}{\lambda_{1}} u_{i} u_{j}\right) e^{-a u}>-C_{1},
$$

${ }^{6}$ Following the usual custom, we shall use the same letter $C$ to represent all absolute constants. No confusion will result from this practice. 
where $C_{1}$ is the constant bounding from below the expression in square brackets in (10). In the case of Hypothesis I, a must be chosen sufficiently large (depending on $\gamma, n, \rho$ and the diameter of $\Omega$ ) so that the quantity in parentheses in (12) is positive, then $m$ chosen so that the product exceeds $-C_{1}$. When Hypothesis II is satisfied, however, the function $u$ depends only on one variable, since the origin of coordinates can be translated to $q$ and the axes rotated so that $u=x_{i}$ for some fixed $i$. Note that the changes of variables caused by translation and rotation do not affect the ellipticity properties of (7) or the constants involved. Hence in this case the first term on the right of (12) vanishes entirely, and the quantity exceeds zero for any $a>0$.

Now $\psi=0$ on $\partial \Omega$ by construction, so (10) and (12) imply

$$
\begin{aligned}
& a_{i j}(\psi+w)_{i j}>0 \text { in } \Omega, \text { and } \\
& \max _{\partial \Omega}(\psi+w)=\max _{\partial \Omega} w=m .
\end{aligned}
$$

Hence $\psi+w$ assumes its maximum $m$ in $\bar{\Omega}$ at $q$, and therefore $[\partial(\psi+w) / \partial y](q) \geqq 0$ for any direction $y$ from $\bar{\Omega}$ into $q$. This gives a lower bound for $\partial \varphi(q) / \partial y$ in terms of the constants $a, \gamma$, $\rho$, etc., involved in the problem, and since $q$ is arbitrary on $\partial \Omega$, this bounds $\partial \varphi / \partial y$ from below on $\partial \Omega$. In a similar way we can obtain an upper bound, which yields the desired estimate on $|\nabla \varphi|$.

In the above proof we chose a constant $C$ so that $|\nabla \varphi|^{\alpha} \leqq$ $C\left(|\nabla \phi|^{2}+1\right)$. Such a $C$ can always be found if $\alpha \leqq 2$, and since this was the only place in the proof of Lemma 1 where $\alpha$ appeared explicitly, we immediately have the following consequence of Lemma 1:

CoRollary 1. Suppose that all the hypotheses of Theorem 1 are satisfied, except that now $G(|p|) / \lambda(|p|) \leqq C|p|^{\alpha}$ with $\alpha \leqq 2$. If there exists a constant $M$ such that $|\varphi| \leqq M$ for any solution $\varphi$ of (7), (8), and if it is known a priori that $|\nabla \varphi|$ assumes its maximum on $\partial \Omega$, then problem (7), (8) has a solution.

For, then $|\nabla \varphi|$ is bounded by the constant of Lemma 1 in $\bar{\Omega}$ and hence Lemma 3 (below) is true, from which existence follows. Similar corollaries hold for Theorems 2 and 3. One very important class of equations in two dimensions for which $|\nabla \phi|^{2}=\varphi_{x}^{2}+\varphi_{y}^{2}$ assumes its maximum on $\partial \Omega$ is the following, having to do with the mean curvature of a surface:

$$
\frac{\partial}{\partial x}\left(\frac{\varphi_{x}}{\sqrt{\left(1+|\nabla \varphi|^{2}\right)}}\right)+\frac{\partial}{\partial y}\left(\frac{\varphi_{y}}{\sqrt{\left(1+|\nabla \varphi|^{2}\right)}}\right)=f\left(1+|\nabla \varphi|^{2}\right),
$$


where $f$ is continuously differentiable and $|f(p)| \leqq C|p|^{\beta}(\beta \leqq-1)$. This is proved in the classical paper of Bernstein [3]. Another simple general example, this time in $n$ dimensions, where Corollary 1 holds is the following:

$$
\Delta \varphi=q\left(\varphi_{x}\right),
$$

where $q$ is a quadratic function.

The task of the next lemma is to demonstrate that $|\nabla \varphi|$ is bounded in the interior of $\Omega$. This is done by showing that it cannot grow more than a fixed amount larger than its maximum on $\partial \Omega$.

LEMma 2. Let the conditions of Lemma 1 be satisfied. Then there is a constant $K=K(C, \alpha, \gamma, n ; \Omega)$ independent of $\phi$ such that $|\nabla \varphi| \leqq K$ throughout $\Omega$, for all solutions $\varphi$ of the problem (7), (8).

Proof. For the purpose of this proof, fix an integer $j$ between 1 and $n$ and let $v=\partial \varphi / \partial x_{j}$. Let $\xi$ be a smooth function of compact support in $\Omega$. Then (7) implies that

$$
\int_{\Omega}\left\{\frac{\partial}{\partial x_{j}}\left[a_{k}\left(\varphi_{x}\right)\right]-g\left(x, \varphi, \varphi_{x}\right)\right\} \xi_{j} d x=0 .
$$

If we now integrate the first term of this expression by parts, moving the $x_{k}$ derivative to $\xi$ and the $x_{j}$ derivative to $a_{k}$, we obtain the expression

$$
\int_{\Omega}\left(a_{k l} \frac{\partial v}{\partial x_{l}} \xi_{k}-g \xi_{j}\right) d x=0 .
$$

By a simple limiting procedure it is easy to see that (13) holds for piecewise differentiable $\xi$ of compact support in $\Omega$. Let $m=\max _{\partial \Omega}|\nabla \phi|$ and $M=\max _{\Omega}|\nabla \varphi|$. If $M \leqq m$, the proof is complete, by Lemma 1 . If $M>m$, it is our task to show that $M$ is independent of any particular choice of $\varphi$, and only depends on $\alpha, m, C$, the volume of $\Omega$, and the number of dimensions. Let us fix a number $h$ between $m$ and $M$ and define

$$
\xi(x)= \begin{cases}v(x)-h & \text { for } v \geqq h \\ 0 & \text { for } v<h .\end{cases}
$$

Clearly $\xi(x)$ is of compact support in $\Omega$, and furthermore is an admissible function for (13), as can be seen by going to the limit in (13). Thus we obtain

$$
\int_{v \geqq h} a_{k l} v_{k} v_{l} d x=\int_{v \geqq h} g v_{j} d x
$$


What follows is not strictly rigorous, since the surface $v=h$ may in principle be very complicated. However, the details will not be pursued, with the note that the alternate method of proof based on Stampacchia's work, which is outlined later, avoids this difficulty. So, proceeding heuristically, we can write (14) in the form

$$
\int_{h}^{M} d v \int_{v=\mathrm{censt}} \frac{\alpha_{k l} v_{k} v_{l}}{|\nabla v|} d S=\int_{h}^{M} d v \int_{v=\mathrm{const}} \frac{g v_{j}}{|\nabla v|} d S
$$

where $d S$ is the surface element of the surface $v=$ constant. Since this must hold for all possible $h$ and $M$, we have

$$
\int_{v=h} \frac{a_{k l} v_{k} v_{l}}{|\nabla v|} d S=\int_{v=h} \frac{g v_{j}}{|\nabla v|} .
$$

By the hypothesis of the lemma and Schwarz's inequality,

$$
\int_{v=h} \lambda\left(\left|\varphi_{x}\right|\right)|\nabla v| d S \leqq \int_{v=h} G\left(\left|\varphi_{x}\right|\right) d S,
$$

or finally, since $v=\varphi_{j}=h$ and $\left(\sum_{i \neq j}\left|\varphi_{i}\right|^{2}\right)^{1 / 2} \leqq M$, we have

$$
\int_{v=h}|\nabla v| d s \leqq \frac{G(h+M)}{\lambda(h+M)} S(h)
$$

where $S(h)$ is the area of the surface where $v=h$. This is the step in which the hypotheses on the monotonicity of $G$ and $\lambda$ are necessary, in order to pull both quantities through the integral sign, preserving the same arguments in each. The present hypotheses on $G$ and $\lambda$ are retained for simplicity, but we remark that they could be generalized to allow $G$ and $\lambda$ to be functions of $\left(p_{1}, p_{2}, \cdots, p_{n}\right)$ instead of $|p|$, such that if $G$ is monotone one way in a given $p_{i}$, $\lambda$ is monotone the opposite way in the same $p_{i}$.

To continue (following Weinberger [15]), if $V(h)$ is the volume of the region where $v \geqq h$, we know that

$$
V^{\prime}(h)=-\int_{v=h}|\nabla v|^{-1} d s \leqq-\frac{S^{2}(h)}{\int|\nabla v| d S}
$$

by Schwarz's inequality; and by the isoperimetric inequality

$$
S(h) \geqq C V(h)^{1-1 / n}
$$

where $C$ is a constant depending only on $n$. Combining (15), (16), and (17), we obtain 


$$
V^{\prime}(h) V(h)^{1 / n-1} \leqq-C \frac{\lambda(h+M)}{G(h+M)}
$$

for a new $C$. Integrating this inequality from $h$ to $k$, we finally have

$$
V(h)^{1 / n}-V(k)^{1 / n} \geqq C \int_{h}^{k} \frac{\lambda(\xi+M)}{G(\xi+M)} d \xi .
$$

This holds in particular for $k=M$, where

$$
V(h)^{1 / n} \geqq C \int_{h}^{M} \frac{\lambda(\xi+M)}{G(\xi+M)} d \xi
$$

since $V(M)=0$. Now the left side is bounded, since $V(h)$ is always no greater than the volume of $\Omega$, which we have assumed finite. By the hypothesis of Theorem 1 (cf. footnote 3), the right side becomes unbounded as $M \rightarrow \infty$. Hence we conclude that $M$ is bounded by a constant depending only on $n, \gamma, \alpha, C$ and the volume of $\Omega$, and $m$, which by Lemma 1 also only depends on $n, \gamma, \alpha, C$ and $\Omega$.

From the crucial inequality of the above lemma

$$
V(h)^{1 / n} \geqq C \int_{h}^{M} \frac{\lambda(\xi+M)}{G(\xi+M)} d \xi,
$$

we see that since the right side is monotone increasing in $M, M$ must be bounded if $\Omega$ has sufficiently small volume, depending on the magnitude of the constants involved. Hence we have

CoRollary 2. Suppose that all the hypotheses of Theorem 1 are satisfied, except that now $G(|p|) / \lambda(|p|) \leqq C|p|^{\alpha}$ with $\alpha \leqq 2$. If there exists a constant $M$ such that $|\varphi| \leqq M$ for any solution of (7), (8), and if the volume of $\Omega$ is sufficiently small as defined by the above inequality, then problem (7), (8) has a solution.

For, as we have seen, Lemma 2 then holds, and hence so does the rest of the proof of Theorem 1. This corollary, and indeed, the entire theory can be generalized in the uniformly elliptic case to include the boundary condition $\varphi=f$ on $\partial \Omega, f$ being twice continuously differentiable; in the nonuniformly elliptic case a similar generalization is possible under the additional assumption that $\Sigma\left|a_{k l}(p)\right| / \lambda_{1}(p) \leqq$ $C\left(|p|^{2}+1\right)$ for some constant $C>0$. In either case only a slight alteration of the proof of Lemma 1 is necessary.

Unfortunately the difficulties in extending the theory of the 
uniformly elliptic case to continuous but nonsmooth boundary conditions are at present unsurmountable without further assumptions (see Akô $[1,2])$, and without such an extension this "existence in the small" cannot be used to prove existence in the large by a method such as the Perron process.

One can construct a second proof of Lemma 2 using a technique of Stampacchia's, as outlined below for the case when $\lambda$ and $G$ are constants. The reader can easily see how the proof can be modified to include the use of variable $\lambda$ and $G$ considered above.

We begin with (14) and obtain the following:

$$
\begin{aligned}
\lambda \int_{v \geqq h}|\nabla v|^{2} d x & \leqq \int_{v \geqq h} a_{i k} v_{i} v_{k} d x \\
& \leqq\left|\int_{v \geqq h} g v_{j} d x\right| \leqq \frac{\lambda}{2} \int|\nabla v|^{2} d x+\frac{1}{2 \lambda} \int|g|^{2} d x,
\end{aligned}
$$

which leads to

$$
\int_{v \geqq h}|\nabla v|^{2} d x \leqq \frac{G^{2}}{\lambda^{2}} V(h) .
$$

Now, by a variation of the Sobolev inequality (see [9]), we obtain for $k>h$ and $1 / q=1 / 2-1 / n$

$$
\begin{aligned}
(k-h)^{2} V(k)^{2 / q} \leqq\left[\int_{v \geqq h}(v-h)^{q} d x\right]^{2 / q} & \leqq\left[\int_{v \geqq h}(v-h)^{q} d x\right]^{2 / q} \\
& \leqq C\left(\int_{v \geqq h}|v \nabla|^{2} d x\right),
\end{aligned}
$$

and hence by combining (18) and (19) we get an inequality of the form

$$
V(k) \leqq \frac{C}{(k-h)^{\alpha}} V(h)^{\beta}
$$

where $\alpha>0$ and $\beta>1$, for all $k>h>m$. By Stampacchia's lemma ([13], pp. 400-401), for any function $V$ satisfying this inequality, there exists a constant $d$ depending on $C, \alpha, \beta$, and hence on the constants of the problem, such that $V(m+d)=0$. This implies that

$$
|v| \leqq m+d
$$

and completes the proof. In the more general case where $\lambda$ and $G$ are not constants, we obtain an inequality of the form

$$
|v| \leqq m+C|v|^{\alpha},
$$

where $\alpha<1$, which still yields a bound of the desired type for $|v|$. 
Lemma 3. Let equation (7) satisfy the conditions of the preceeding lemmas. Then there exist constants $D$ and a depending only on $C, \alpha, \gamma, n$ and $\Omega$, independent of $\varphi$, such that

$$
|\nabla \varphi(x)-\nabla \varphi(y)| \leqq D|x-y|^{a}
$$

for all solutions $\varnothing$ of (7), (8), and all $x, y \in \Omega$.

Proof. Since by Lemma $2,|\nabla \phi| \leqq K(C, \alpha, \gamma, n ; \Omega), \lambda_{1}\left(\varphi_{x}\right)$ is bounded from above and below (away from zero); this plus the boundedness of $|\varphi|$ and continuity ensure that the $a_{i j}$ and $g$ are also bounded by constants. In addition we assume that in a neighborhood of each boundary point $q \in \partial \Omega$ there is a twice continuously differentiable one-to-one transformation $x \rightarrow y$ that takes the surface element of $\partial \Omega$ at $q$ into a plane element $y_{n}=0$. This is what we mean by the assumption that $\partial \Omega$ is "sufficiently smooth." These facts imply the required result, by a generalized version of de Giorgi's theorem ([7], p. 56, Theorem 11) which states that under these conditions the Hölder continuity of $|\nabla \varphi|$ can be estimated in terms of $\max |\varphi|, \max |\nabla \phi|$, $\max \left|a_{i j}\right|, \max |g|$, and $\min \left|\lambda_{1}\right|$. Each of these quantities, by the preceding work, depends on $\alpha, \gamma, C, n$, and $\Omega$, and not on any choice of $\varphi$. This completes the proof.

Having now proved these three lemmas, we can proceed to complete the proof of Theorem 1. This proof is by a relatively standard technique, so we shall be brief. We shall assume that $g$ and the $a_{k l}$ are Lipschitz continuous in all arguments, with the understanding that once existence is proved, we can obtain existence when $g$ and the $a_{k l}$ are Hölder continuous by applying the Schauder theory to a sequence of equations with Lipschitz continuous coefficients which coverage to the given equation, and passing to the limit.

Let $\mathscr{S}$ be the Banach space of functions $\varphi$ which are differentiable and whose gradients are Hölder continuous with exponent $a$, where $a$ is the number determined in Lemma 3. The norm on $\mathscr{S}$ is

$$
\|\varphi\|=\operatorname{lub}_{\Omega}|\varphi|+\operatorname{lub}_{\Omega}|\nabla \varphi|+\operatorname{lub}_{\Omega} \frac{|\nabla \varphi(x)-\varphi(y)|}{|x-y|^{a}} .
$$

Now we choose a function $\varphi \in \mathscr{S}$ and place it and its appropriate partial derivatives in the coefficients of (7); setting

$$
A_{i k}(x)=\frac{a_{i k}\left(\varphi_{x}\right)+a_{k i}\left(\varphi_{x}\right)}{2}
$$

and $G(x)=g\left(x, \varphi, \varphi_{x}\right)$ we obtain a uniformly elliptic linear equation 


$$
A_{i k}(x) \frac{\partial^{2} \psi}{\partial x_{i} \partial x_{k}}=G(x)
$$

The solution is of this equation with zero boundary values exists by the Schauder theory of linear equations (see [5], pp. $331 \mathrm{ff}$ ), and Lemmas 1,2 , and 3 show that $\psi \in \mathscr{S}$. Thus we have defined a mapping $\varphi \rightarrow T \varphi=\psi$ of $\mathscr{S}$ onto itself; the complete boundary value problem (7), (8) has a solution if there is a $\varphi$ such that $\varphi=T \varphi$.

Consider the family of equations

$$
\varphi-\sigma T \varphi=0, \quad 0 \leqq \sigma \leqq 1 .
$$

We now note that for each $\sigma \in[0,1]$, a solution $\varphi(x ; \sigma)$ of $(20)$ would coincide with a solution of (7), (8) where the right side is replaced by $\sigma g$. Thus the inequalities of our lemmas hold uniformly for $\sigma \in[0,1]$, and we can conclude that solutions of (20), when they exist, are uniformly bounded in $\mathscr{S}$ independent of $\sigma$, i.e., $\|\varphi(x ; \sigma)\| \leqq C$ where $C$ does not depend on $\sigma$. The Leray-Schauder fixed-point theorem in the form due to Schaefer [12] asserts that if the latter is true and if $T$ is a completely continuous transformation of a linear, complete, locally convex Hausdorff space into itself, then (20) has a solution for each $\sigma \in[0,1]$. Since the complete continuity of $T$ follows from the classical Schauder theory ([5], pp. $331 \mathrm{ff})$ and $\mathscr{S}$ is a Banach space, the proof of Theorem 1 is complete.

The proofs of Theorems 2 and 3 are almost identical with that of Theorem 1, $g$ being replaced and augmented, respectively, by the derivatives of the $\alpha_{k}$ with respect to the $x$ variables. It is now also clear how the corollaries follow from the theorems and the lemmas of this section.

4. On the necessity of certain of the hypotheses. We proved Theorem 1 under two hypotheses, namely, uniform ellipticity and nonuniform ellipticity. In the latter case part of the hypothesis was that $\Omega$ be strictly convex. That some convexity condition is necessary to prove an existence theorem in the nonuniformly elliptic case is illustrated by the following example in two variables:

$$
\begin{aligned}
& \frac{\partial}{\partial x}\left(\frac{\psi_{x}+f_{x}}{\sqrt{\left(1+\mid \bar{V}[+f]^{2}\right)}}\right)+\frac{\partial}{\partial y}\left(\frac{\psi_{y}+f_{y}}{\sqrt{\left(1+|\nabla\lfloor\psi+f]|^{2}\right)}}\right) \\
& \quad=0 \text { in } \Omega, \gamma=0 \text { on } \partial \Omega
\end{aligned}
$$

where $\psi$ is the unknown function and $f$ is a smooth function given in $\bar{\Omega}$. A routine calculation serves to verify that this equation is nonuniformly elliptic. But if we set $\varphi=\psi-f$, this is equivalent to 
$\frac{\partial}{\partial x}\left(\frac{\varphi_{x}}{\sqrt{\left(1+|\nabla \phi|^{2}\right)}}\right)+\frac{\partial}{\partial y}\left(\frac{\varphi_{y}}{\sqrt{\left(1+|\nabla \varphi|^{2}\right)}}\right)=0$ in $\Omega, \varphi=f$ on $\partial \Omega$,

the well-known minimal surface equation, which in general has no solution unless $\Omega$ is convex.

In Lemma 1 we used the strict convexity of $\Omega$ (or the boundedness of $\left.\lambda_{2} / \lambda_{1}\right)$ to prove the boundedness of $|\nabla \varphi|$ on $\partial \Omega$. In the nonuniformly elliptic case we required the assistance of a function which measured the distance from a point $x$ of $\Omega$ to the plane intersecting $\partial \Omega$ at some point $q$. Of course if $\Omega$ were convex but not strictly convex, that is, if $\partial \Omega$ has a "flat" portion, the same argument would suffice. The only essential thing is that the plane in question should intersect $\bar{\Omega}$ only in points of $\partial \Omega$. This is still possible if $\Omega$ is convex, and hence the adverb "strictly" can be stricken from the wording of Hypothesis II. One should bear in mind, however, that we are considering solutions which vanish on $\partial \Omega$. If nonzero boundary values are allowed, then strict convexity is required.

Next we shall consider the necessity that we have $\alpha<1$ in the inequality

$$
\frac{G(|p|)}{\lambda(|p|)} \leqq C|p|^{\alpha}
$$

of Theorem 1. As we saw in $\S 3$, Theorem 1 holds for $\alpha \leqq 2$ provided we know a priori that $|\nabla \varphi|$ assumes its maximum on $\partial \Omega$. We have analogous results for Theorems 2 and 3. And Corollary 2 shows that "existence in the small," i.e., existence in small enough domains, is guaranteed if the boundary values are smooth and if $\alpha \leqq 2$. Another result is the following:

THEOREM 4. Let us consider the problem

$$
\frac{\partial}{\partial x_{k}}\left[a_{k}\left(x, \varphi_{x}\right)\right]=0 \text { in } \Omega, \quad \varphi=0 \text { on } \partial \Omega,
$$

where the hypotheses of Theorem 2 are satisfied, except that how $A(|p|) / \lambda(|p|) \leqq C|p|^{\alpha}$ with $\alpha \leqq 2$. If in addition it is known that there is a constant $K$ such that $|\varphi| \leqq K$ for all solutions $\varphi$ of problem (21), and that the $a_{k l}$ are differentiable such that for sufficiently large $|p|$ there are constants $C_{i}$ with

$$
\sum_{i, j, k}\left|\frac{\partial a_{i j}}{\partial p_{k}}\right| \leqq C_{1} \frac{\lambda_{1}}{|p|}, \sum_{i, j, k}\left|\frac{\partial a_{i j}}{\partial x_{k}}\right| \leqq C_{2} \lambda_{1}, \sum_{i, j, k}\left|\frac{\partial a_{k}}{\partial x_{i} \partial x_{j}}\right| \leqq C_{3} \lambda_{1}|p|^{2},
$$

then a solution to problem (21) exists. 
For, if the above hypotheses are true, one can prove that $|\nabla \phi|$ is bounded throughout $\Omega$ using the techniques of LadyzhenskayaUral'tseva ([7], pp. $41 \mathrm{ff}$; esp. p. 45). Our hypotheses are slightly more general than theirs, but it is clear how their proofs can be modified. From the boundedness of $|\nabla \phi|$ in $\Omega$, Lemma 3 and existence follow immediately.

The following result, due to Akô [1], is also significant:

TheOrem 4. Consider the elliptic equation

$$
\left.a_{k l}(x) \varphi_{k l}=g\left(x, \varphi, \varphi_{x}\right) \text {, with } \varphi=f \text { on } \partial \Omega \text { ( } f \text { continuous }\right) \text {. }
$$

If $|g(x, \varphi, p)| \leqq C_{1}(M) p+C_{2}(M)$ when $|\varphi| \leqq M$, and $g$ is nondecreasing in $\varphi$ for all $x$ and $p$, then this problem has a solution, which is unique if $g$ satisfies an Osgood condition in the $p$ variables.

Akô has also recently shown [2] that Theorem 3 holds in the uniformly elliptic case for $\alpha=1$ provided that $\gamma$ (the bound on the ratio of $\lambda_{2}$ to $\lambda_{1}$ ) is sufficiently near 1 . Other than these results the case $\alpha=1$ is still open. Certainly if in general Theorem 3 is not true for $\alpha=1$, counterexamples will be hard to come by, thanks to the results of Akô.

However, we can definitely state that for $\alpha>1$, Theorem 1 is not in general true. The following example in two dimensions will serve to illustrate this:

$$
\begin{aligned}
\varphi_{x x}+\varphi_{y y} & =\left\{\begin{array}{l}
1 \text { for } \varphi_{x}^{2}+\varphi_{y}^{2} \leqq 1 \\
\left(\varphi_{x}^{2}+\varphi_{y}^{2}\right)^{\alpha / 2} \text { for } \varphi_{x}^{2}+\varphi_{y}^{2}>1
\end{array} \text { in } \Omega(1<\alpha<2),\right. \\
\varphi & =0 \text { on } \partial \Omega .
\end{aligned}
$$

It is clear that this equation satisfies all the conditions of Theorem 1 ; in particular, the right side is Hölder continuous. We shall take $\Omega$ to be a circle in the $(x, y)$ plane with center at the origin and radius

$$
R>R_{0}=\left(\frac{\alpha}{\alpha-1} 2^{1-\alpha}\right)^{1 /(2-\alpha)} .
$$

In such a region with radial symmetry and boundary values which depend only on $r$, a solution of (22) must be a function of $r$ alone, since the equation itself is invariant under rotation of coordinates. In fact, if we convert (22) to polar coordinates, we obtain the two equations

$$
\left(r \psi_{1}^{\prime}\right)^{\prime}=r \text { for }\left|\psi_{1}^{\prime}\right| \leqq 1
$$




$$
\left(r \psi_{2}^{\prime}\right)^{\prime}=r\left|\psi_{2}^{\prime}\right|^{\alpha} \text { for }\left|\psi_{2}^{\prime}\right|>1
$$

where the prime denotes differentiation with respect to $r$. Because of the symmetry of the problem, one of these equations must hold in a circle around the origin, and then, at worst, the equations hold alternately in concentric annular regions. Integrating these equations once, we obtain $\psi_{1}^{\prime}$ and $\psi_{2}^{\prime}$ as functions of $r$ :

$$
\psi_{1}^{\prime}=\frac{r}{2}+\frac{C_{1}}{2} ; \psi_{2}^{\prime}=\frac{1}{r}\left[\frac{1-\alpha}{2-\alpha}\left(r^{2-\alpha}+C_{2}\right)\right]^{1 /(1-\alpha)}
$$

where $C_{1}$ and $C_{2}$ are arbitrary constants to be chosen so that $\psi_{1}^{\prime}$ and $\psi_{2}^{\prime}$ are defined in their respective regions and match on the boundary or boundaries between.

By examining these functions, we see that $\psi_{2}^{\prime}$ is never defined at the origin. $\psi_{1}^{\prime}$ is, provided that $C_{1}=0$. Hence $\psi_{1}$ is the solution for $0 \leqq r \leqq 2$. For $r>2, \psi_{1}^{\prime}>1$ and hence $\psi_{2}$ must be the solution. Since we must have $\psi_{1}^{\prime}(2)=\psi_{2}^{\prime}(2)$, we compute $C_{2}=2^{1-\alpha}(\alpha / 1-\alpha)$. By examining $\psi_{2}^{\prime \prime}$, we see that $\psi_{2}^{\prime}$ is monotone increasing in $r$ for $r>\left(\alpha 2^{1-\alpha}\right)^{1 /(2-\alpha)}$, hence for $r>2$, and approaches infinity as $r \rightarrow R_{0}=\left(-C_{2}\right)^{1 /(2-\alpha)}$. For $r \geqq R_{0}, \psi_{2}^{\prime}$ is not defined.

We must therefore conclude that no solution exists in a circle of radius $R \geqq R_{0}$. Note, however, that a solution does exist in a circle of radius $R<R_{0}$, which agrees with our comments that a solution is always possible for $\alpha \leqq 2$ provided that the domain $\Omega$ is small enough.

The above example also serves to illustrate that Theorem 3 does not in general hold for $\alpha>1$. For Theorem 2, the same example suffices, but in somewhat modified form:

$$
\begin{gathered}
\frac{\partial}{\partial x}\left[\varphi_{x}+\varphi_{y}-\frac{e^{2 x}+e^{2 y}}{2}\right]+\frac{\partial}{\partial y}\left[\varphi_{x}+\varphi_{y}-\frac{e^{2 x}+e^{2 y}}{2}\right]=0 \\
\text { for } e^{-2 x} \varphi_{x}^{2}+e^{-2 y} \varphi_{1}^{2} \leqq 1 ; \\
\frac{\partial}{\partial x}\left[\frac{\left(\varphi_{x}+\varphi_{y}\right)^{1-\alpha}}{1-\alpha}-\frac{\left(e^{2 x}+e^{2 y}\right)^{1-\alpha / 2}}{2-\alpha}\right] \\
\quad+\frac{\partial}{\partial y}\left[\frac{\left(\varphi_{x}+\varphi_{y}\right)^{1-\alpha}}{1-\alpha}-\frac{\left(e^{2 x}+e^{2 y}\right)^{1-\alpha / 2}}{2-\alpha}\right]=0 \\
\text { for } e^{-2 x} \varphi_{x}^{2}+e^{-2 y} \varphi_{y}^{2}>1,
\end{gathered}
$$

with $1<\alpha<2$ and where $\Omega$ is the domain $e^{2 x}+e^{2 y}=R^{2} \geqq R_{0}^{2}, R_{0}$ being the same constant as before. By making the substitution $x^{\prime}=$ $e^{x}, y^{\prime}=e^{y}$ in the above and noting that the new equation has radial symmetry in the $\left(x^{\prime}, y^{\prime}\right)$ plane, and hence that the solution is a function 
of $r=\left[\left(x^{\prime}\right)^{2}+\left(y^{\prime}\right)^{2}\right]^{1 / 2}$ alone provided the boundary values are zero or constant, one can see that the above reduces to the same equation as was considered in connection with Theorems 1 and 3 . Hence the same conclusion holds: namely, that no solution exists if $R \geqq R_{0}$.

5. Conditions on the inhomogeneous term. Now, finally we shall explore some conditions on the inhomogeneous term $g\left(x, \varphi, \varphi_{x}\right)$ sufficient to establish the existence of an a priori bound on $|\varphi|$, which will show that there is a function $G(|p|) \geqq|g(x, \varnothing, p)|$ satisfying the hypotheses of Theorem 1.

1. If there is a constant $M$ such that $\varphi g(x, \varphi, p)>0$ for all $|\varphi|>M$, uniformly in $x$ and $p$, then $|\varphi| \leqq M$. For if $\varphi$ assumes a positive maximum exceeding $M$ at a point $x_{0}$, then at $x_{0}$ we have

$$
0 \geqq \alpha_{i j} \varphi_{i j} \varphi=\varphi g\left(x, \varphi, \varphi_{x}\right)>0,
$$

an obvious absurdity. The case of a negative minimum is treated similarly. Note that in the nonuniformly elliptic case, when $g$ does not depend on $\varphi_{x}$, this condition is a special case of Stampacchia's ([14]; see also $6^{\circ}$ below). Example: $g(\varphi)=\varphi-2 \sin \varphi+\varepsilon$. (The small constant $\varepsilon$ is added to eliminate the possibility of the trivial case $\varphi \equiv 0)$.

2. ${ }^{\circ}$ Suppose there exists a constant $M$ independent of $x$ and $p$ such that for each fixed $x$ and $p, g(x, \pm M, p)=0$. Then it is clear that we can alter the definition of $g$ for $|\varphi|>M$ so that $\varphi g>0$ for $|\varphi|>M$. One can then prove the existence of a solution to (3), (4) bounded in absolute value by $M$, using condition $1^{\circ}$. But this is also a solution of the current problem since for $|\varphi| \leqq M$, the original $g$ and the redefined $g$ coincide.

Example: $g=\varphi \sin \varphi+\varepsilon$.

$3 .^{\circ}$ Let a constant $\beta>0$ exist such that $\int_{0}^{1} g_{\varphi}(x, t \varphi, t p) d t \geqq \beta$ uniformly in $x, \varphi$ and $p$. Then by examining the linear equation for $\varphi$

$$
a_{k l} \varphi_{k l}=\varphi_{k} \int_{0}^{1} g_{\phi_{k}}\left(x, t \varphi, t \varphi_{x}\right) d t+\varphi \int_{0}^{1} g_{\varphi}\left(x, t \varphi, t \varphi_{x}\right) d t+g(x, 0,0)
$$

obtained by replacing

$$
g(x, \varphi, p) \text { by } \int_{0}^{1} \frac{d g(x, t \varphi, t p)}{d t} d t+g(x, 0,0),
$$


at points where $\varphi$ attains a positive maximum and negative minimum, we easily see that

$$
|\varphi| \leqq \frac{\max |g(x, 0,0)|}{\beta} \text {. Example: } g(\varphi)=\left\{\begin{aligned}
-\frac{1}{\varphi+1} \text { for } \varphi \geqq 0 \\
\varphi-1 \text { for } \varphi<0 .
\end{aligned}\right.
$$

4. Suppose there exist constants $\lambda>0, k>0$, and functions $h(x, \varphi, p)$ and $C(x, p)$, and an integer $i$ between 1 and $n$, such that $a_{i i}(p)>\lambda, g(x, \varphi, p)=C(x, p) \varphi+h(x, \varphi, p)$, where $h$ is bounded and $C(x, p) \geqq-k$; and suppose $\Omega$ is small enough so that its diameter $d$ satisfies $|\exp (d / \sqrt{\lambda})-1|<1 / k$. Then

$$
|\varphi| \leqq \frac{(\exp [d / \sqrt{\lambda}]-1) \max |h(x, \varphi, p)|}{1-k(\exp [d / \sqrt{\lambda}]-1)}
$$

In fact, this even gives a bound on $|\varphi|$ if there exist two constants $C$ and $\alpha<1$ such that $|h(x, \varphi, p)|<C|\varphi|^{\alpha}$. This bound is a generalization of a result known for linear equations (see [4]). Example of an equation in three variables where this holds:

$$
\varphi_{x x}+\frac{\partial}{\partial y}\left[\alpha_{2}(\nabla \varphi)\right]+\frac{\partial}{\partial z}\left[\alpha_{3}(\nabla \varphi)\right]=f(x)-\frac{1}{26} \varphi
$$

where $\Omega$ is the cube $0<|x|,|y|,|z|<\pi$ and where $a_{1}=\varphi_{x}, a_{2}$, and $a_{3}$ satisfy Hypothesis I or II.

This list gives an idea of the conditions on $g(x, \varphi, p)$ under which $|\varphi|$ is uniformly bounded. That some such conditions are necessary is illustrated by the equation in three variables

$$
\Delta \varphi=f(x)-3 \varphi
$$

where $\Omega$ is the cube of $4^{\circ}$ above. It is well known that for arbitrary $f(x)$, this equation does not in general have a solution. Note that the given $g(x, \varphi)=f(x)-3 \varphi$ satisfies none of conditions $1^{\circ}-4^{\circ}$.

If $g$ is a function of $x$ and $\varphi$ alone, as in the corollary following Theorem 1 , it is possible to restate conditions $1^{\circ}-4^{\circ}$, removing any of the statements regarding dependence on $\Phi_{x}$. Since this is a simple exercise, it will not be carried through here. It should be instructive, however, to state two new conditions which will suffice to give an a priori bound on $\varphi$ when $g$ does not depend on $\varphi_{x}$.

5. This is a variation of condition $4^{\circ}$, and illustrates how such statements can often be improved by removing dependence on $\varphi_{x}$. 


$$
g(x, \varphi)=\varphi \int_{0}^{1} g_{\varphi}(x, t \varphi) d t+g(x, 0) .
$$

Hence if $\int_{0}^{1} g_{\varphi}(x, t \varphi) d t \geqq-k$ for all $x$ and $\varphi, k$ being a positive constant, and if the other conditions are as in $4^{\circ}$, we can obtain a bound on $|\varphi|$ of the same form.

6. If there exists a function $f(p)$ such that for each $k$,

$$
a_{k}(p)=\frac{\partial f}{\partial p_{k}},
$$

and Hypothesis II (non-uniform ellipticity) is satisfied with $\lambda_{1}(p)=$ $\nu\left(1+|p|^{2}\right)^{\tau}\left(\nu>0,-\frac{1}{2}<\tau\right)$ and $g(x, \varphi)$ is such that there exist constants $K>0, R$, and $M$ with

$$
\begin{aligned}
& g(x, \varphi) \geqq-\alpha q|\varphi|^{\alpha-1}-R \text { if } \varphi \geqq K>0, \\
& g(x, \varphi) \leqq M|\varphi|^{\beta} \text { if } \varphi \leqq-K<0,
\end{aligned}
$$

where $\alpha=2(\tau+1)>1, \beta<(\alpha n / \alpha-n)-1$, and

$$
q<[\nu / \alpha] \inf _{\{\varphi: \varphi=0 \text { on } \partial \Omega\}} \frac{\int_{\Omega}|\nabla \varphi|^{\alpha} d x}{\int_{\Omega}|\varphi|^{\alpha} d x},
$$

then an a priori bound for $\varphi$ exists. This result is due to Stampacchia [14]. As long as we are considering the nonuniformly elliptic case, this condition includes $1^{\circ}$ as a special case when $g$ does not depend on $\varphi_{x}$. An example in three dimensions of an equation satisfying this condition but not $1^{\circ}$ is

$$
\Delta \phi=f(x)-\frac{9 \pi}{10} \varphi
$$

where $\Omega$ is the cube of $4^{\circ}$.

The author wishes to thank Professor David Gilbarg for his help and inspiration.

\section{BIBLIOGRAPHY}

1. K. Akô, On Perron's process associated with second order elliptic differential equations, J. Fac. Sci. Univ. Tokyo Sect. I, 9(1962), 165-202.

2. - Subfunctions for quasilinear elliptic differential equations, J. Fac. Sci. Univ. Tokyo Sect. I 9 (1963), 403-416.

3. S. Bernstein, Sur les surfaces définies au moyen de leur courbure moyenne ou totale, Ann. Ecole Norm. Sup. 27 (1910), 233-256.

4. L. Bers, F. John, and M. Schechter, Lectures in Applied Mathematics, Volume III: Partial Differential Equations, Interscience, New York, pp. 153-154. 
5. R. Courant, and D. Hilbert, Methods of Mathematical Physics, vol. II, Interscience, New York, 1962.

6. D. Gilbarg, Boundary value problems for nonlinear elliptic equations in $n$ variables, Nonlinear Problems, University of Wisconsin Press, Madison, (1963), 151-9.

7. O. Ladyzhenskaya, and N. Ural'tseva, Quasi-linear elliptic equations and variational problems with many independent variables, Russian Math. Surveys 16 No. 1 (1961), 17-91.

8. C. Morrey, Existence and differentiability theorems for variational problems for multiple integrals, Partial Differential Equations and Continuum Mechanics, University of Wisconsin Press, Madison, (1961), 241-270.

9. L. Nirenberg, On elliptic partial difjerential equations, Ann. Scuola Norm. Super. Pisa Ser. 313 (1959), 113-159.

10. R. Redheffer, An extension of certain maximum principles, Monatsch. f. Math. 66 (1962), 32-42.

11. - Eindeutigkeitssätze bei nichtlinearen Differential-gleichungen, J. Reine Angew. Math. 211 (1962), 70-77.

12. H. Schaefer, Über die Methode der a priori Schranken, Math. Ann. 129 (1955), 415-416.

13. G. Stampacchia, Régularisation des solutions de problèmes aux limites elliptiques à données discontinues, International Symposium on Linear Spaces, Jerusalem, (1960), 399-408.

14. - On some regular multiple integral problems in the calculus of variations, Comm. Pure Appl. Math. 16 (1963), 383-421.

15. H. Weinberger, Symmetrization in uniformly elliptic problems, Studies in Mathematical Analysis and Related Topics, Stanford University Press, Stanford, (1962), 424-428.

UNIVERSITY OF CALIFORNIA

Los Alamos Scientific Laboratory 



\section{PACIFIC JOURNAL OF MATHEMATICS}

\section{EDITORS}

\author{
H. SAMELSON \\ Stanford University \\ Stanford, California \\ R. M. BLUMENTHAL \\ University of Washington \\ Seattle, Washington 98105
}

\author{
*J. DUGUNDJI \\ University of Southern California \\ Los Angeles, California 90007
}

RICHARD ARENS

University of California Los Angeles, California 90024

\section{ASSOCIATE EDITORS}
E. F. BECKENBACH
B. H. NEUMANN
F. WOLF
K. YoSIDA

\section{SUPPORTING INSTITUTIONS}

\author{
UNIVERSITY OF BRITISH COLUMBIA \\ CALIFORNIA INSTITUTE OF TECHNOLOGY \\ UNIVERSITY OF CALIFORNIA \\ MONTANA STATE UNIVERSITY \\ UNIVERSITY OF NEVADA \\ NEW MEXICO STATE UNIVERSITY \\ OREGON STATE UNIVERSITY \\ UNIVERSITY OF OREGON \\ OSAKA UNIVERSITY \\ UNIVERSITY OF SOUTHERN CALIFORNIA
}

\author{
STANFORD UNIVERSITY \\ UNIVERSITY OF TOKYO \\ UNIVERSITY OF UTAH \\ WASHINGTON STATE UNIVERSITY \\ UNIVERSITY OF WASHINGTON \\ * * * * \\ AMERICAN MATHEMATICAL SOCIETY \\ CHEVRON RESEARCH CORPORATION \\ TRW SYSTEMS \\ NAVAL ORDNANCE TEST STATION
}

Mathematical papers intended for publication in the Pacific Journal of Mathematics should be typewritten (double spaced). The first paragraph or two must be capable of being used separately as a synopsis of the entire paper. It should not contain references to the bibliography. Manuscripts may be sent to any one of the four editors. All other communications to the editors should be addressed to the managing editor, Richard Arens at the University of California, Los Angeles, California 90024.

50 reprints per author of each article are furnished free of charge; additional copies may be obtained at cost in multiples of 50 .

The Pacific Journal of Mathematics is published monthly. Effective with Volume 16 the price per volume (3 numbers) is $\$ 8.00$; single issues, $\$ 3.00$. Special price for current issues to individual faculty members of supporting institutions and to individual members of the American Mathematical Society: $\$ 4.00$ per volume; single issues $\$ 1.50$. Back numbers are available.

Subscriptions, orders for back numbers, and changes of address should be sent to Pacific Journal of Mathematics, 103 Highland Boulevard, Berkeley 8, California.

Printed at Kokusai Bunken Insatsusha (International Academic Printing Co., Ltd.), No. 6, 2-chome, Fujimi-cho, Chiyoda-ku, Tokyo, Japan.

\section{PUBLISHED BY PACIFIC JOURNAL OF MATHEMATICS, A NON-PROFIT CORPORATION}

The Supporting Institutions listed above contribute to the cost of publication of this Journal, but they are not owners or publishers and have no responsibility for its content or policies.

* Paul A. White, Acting Editor until J. Dugundji returns. 


\section{Pacific Journal of Mathematics}

\section{Vol. 17, No. 2 \\ February, 1966}

Henry A. Antosiewicz, Boundary value problems for nonlinear ordinary

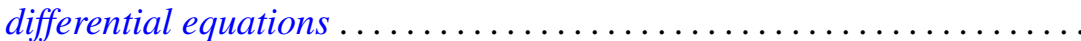

Bernard Werner Levinger and Richard Steven Varga, Minimal Gerschgorin sets. II ........................................

Paul Camion and Alan Jerome Hoffman, On the nonsingularity of complex matrices........................................ 211

J. Chidambaraswamy, Divisibility properties of certain factorials ........ 215

J. Chidambaraswamy, A problem complementary to a problem of Erdös .... 227

John Dauns, Chains of modules with completely reducible quotients ...... 235

Wallace E. Johnson, Existence of half-trajectories in prescribed regions and asymptotic orbital stability ............................ 243

Victor Klee, Paths on polyhedra. II . ........................ 249

Edwin Haena Mookini, Sufficient conditions for an optimal control problem in the calculus of variations ............................ 263

Zane Clinton Motteler, Existence theorems for certain quasi-linear elliptic equations........................................... 279

David Lewis Outcalt, Simple n-associative rings ............... 301

David Joseph Rodabaugh, Some new results on simple algebras ......... 311

Oscar S. Rothaus, Asymptotic properties of groups generation ........... 319

Ernest Edward Shult, Nilpotence of the commutator subgroup in groups admitting fixed point free operator groups .............

William Hall Sills, On absolutely continuous functions and the

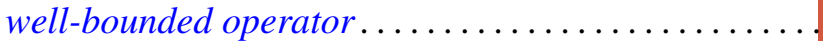

Joseph Gail Stampfli, Which weighted shifts are subnormal ..

Donald Reginald Traylor, Metrizability and completeness in normal Moore spaces 